\title{
Land CSEM simulations and experimental test using metallic casing in a geothermal exploration context: Vallès Basin (NE Spain) case study
}

\author{
Octavio Castillo-Reyes, Pilar Queralt, Alex Marcuello, Juanjo Ledo
}

\begin{abstract}
Controlled-source electromagnetic (CSEM) measurements are complementary data for magnetotelluric (MT) characterization, although its methodology on land is not sufficiently developed and tested as in marine environments. Acquiring expertise in CSEM is crucial for surveys in places where MT cannot be performed due to high-levels of cultural noise. To acquire that expertise, we perform CSEM experiments in the Vallès fault (Northeast (NE), Spain) where MT results have been satisfactory and allow us to verify the CSEM results. The Vallès basin is relevant for potential heat generation because of the presence of several geothermal anomalies, and its nearby location to urban areas. In this paper, we present the experimental setup for that region, a 2-D joint MT+CSEM inverse model, several 3-D CSEM simulations in the presence of metallic casing, and its comparison with real data measurements. We employ a parallel and high-order vector finite element algorithm to discretize the governing equations. By using an adapted meshing strategy, different scenarios are simulated to study the influence of the source position/direction and the conductivity model in a metallic casing presence. An excellent agreement between simulated data and analytical/real field data demonstrates the feasibility of study metallic structures in realistic configurations. Our numerical results confirm that metallic casing strongly influences electromagnetic responses, making surface measurements more sensitive to resistivity variations near the metallic structure. It could be beneficial getting higher signal-to-noise ratios and sensitivity to deep targets. However, such casing effect depends on the input model (e.g., conductivity contrasts, frequency, and geometry).
\end{abstract}

Index Terms-Controlled-source electromagnetic (CSEM), numerical modeling, metallic casing effects, high-performance computing, geothermal exploration.

\section{INTRODUCTION}

O VER the last 20 years, electromagnetic (EM) methods have become invaluable research tools in geophysics with rapidly increasing applications in both industry and academia. Both active and passive EM methods can improve the characterization and interpretation of geophysical datasets by mapping conductivity variations, and reducing ambiguities during exploration surveys. In this sense, the CSEM has become more and more popular in many different application scenarios due to its ability to display conductivity contrasts with respect to their surrounding sediments (e.g., detecting resistive zones in a conductive background). As a result,

Octavio Castillo-Reyes is with Barcelona Supercomputing Center (BSC), Nexus II Building c/Jordi Girona 29, 08034, Barcelona (Spain),

Tel.: +34934137992

octavio.castillo@bsc.es (corresponding author)

Pilar Queralt, Alex Marcuello and Juanjo Ledo are with

Institut Geomodels, Departament de Dinàmica de la Terra i de 1'Oceà

Universitat de Barcelona, 08028 Barcelona, Spain nowadays CSEM has real application in many contexts such as hydrocarbon exploration [1]-[10], reservoir monitoring [11], [12], $\mathrm{CO}_{2}$ storage characterization [13]-[18], and geothermal reservoir imaging [19]-[22], among others.

In marine exploration contexts, CSEM plays a fundamental role in the geological characterization of faults and reservoirs, and allow monitoring the latter due to its high sensitivity to fluid movements and to useful buried resources. In these scenarios, CSEM is a well established supplementing technique to MT [23]-[25]. Also, marine CSEM has provided valuable complementary information to seismic imaging, and has been also used for setting up seismic inversions. However, in land-based exploration environments, CSEM applications for imaging conductivity contrasts among targets and neighbouring materials are scarce, since CSEM needs very different data acquisition and processing strategies [26], [27]. Usually, regions of interest are urbanized and industrialized areas (e.g., railways, power grid, telephone networks, and industrial facilities). Consequently, human-generated noise prevents passive methods such as MT, which is a strong limitation for relevant land-based applications, such as $\mathrm{CO}_{2}$ storage and geothermal exploration. Numerical simulation tools allow us to reproduce different materials' responses to external excitation to analyze observed data and infer models of the subsurface as correct as possible. These simulations have been proven to validate geological models by direct comparison between data and synthetics in different application fields. In the case of land CSEM, these modeling codes are essential to understand the effect of metallic infrastructures and to study their use to improving the signal-to-noise ratio. The analysis of these effects has gained traction recently, and many different approaches have been evaluated on different application contexts. Out of these applications, studies in the area of energy reservoir modeling [28]-[33], water flooding [34], [35], geological storage [36]-[38], geothermal exploration [39], [40], and fractures and fault zones [41], [42], stand out. Regardless of numerical methodology or application area, these works stress out the significant effects on EM responses generated by the presence of steel-cased wells and other metallic infrastructure. Also, authors affirm that CSEM modeling in the presence of metallic structures present a numerically challenging problem because of two main reasons. Firstly, the considerable conductivity contrast between surrounding media and metallic casing. Secondly, the large-scale variation of target bodies requires different resolution levels of discretization. Consequently, simulation 
tools require efficient meshing approaches to incorporate these metallic structures into the modeling.

Gaining experience in 3-D CSEM is crucial to exploring useful underground resources in various environments (e.g., marine and land contexts). This know-how is fundamental for future studies where the MT approach cannot be performed due to high cultural noise levels. To acquire this experience, in this paper, we study the metallic casing effect in a 3-D land CSEM configuration at the Vallès fault (Barcelona, Spain), where previous MT results were satisfactory and allow us to verify, control, and restrict our CSEM results in this region. Several geothermal anomalies have been identified/observed in the Vallès region, and different geophysical surveys have been carried out to characterize its deeper reservoirs. However, the setting remains poorly understood and primarily untapped. The granite bedrock with a highly fractured nature represents a significant challenge for its geological study and numerical modeling. At the same time, its potential for heat generation has brought a tremendous interest, with a nearby location to urban areas. For these reasons, the GEO-URBAN project (ERANET Cofund GEOTHERMICA Project no. 731117) has considered the Vallès region as a test-site, to study and improve different novel geophysical techniques, such as CSEM and passive seismic methods [43].

In the GEO-URBAN scope, we carried out several 3-D land CSEM experiments in the Vallès basin to study a surfaceto-surface CSEM profile and different source-receiver configurations using steel-cased wells to improve the sensitivity and quality of EM measurements. To compute synthetic EM responses, we have used the PETGEM code [8], which has proven to be a flexible, accurate, and efficient large-scale modeling tool on cutting-edge high-performance computing (HPC) architectures. Section II describes the PETGEM mathematical background and details about its computational implementation. In Section III, we present the geophysical acquisition setup for the Vallès basin and its resulting experimental CSEM configuration. In Section IV, we perform PETGEM simulations for different resistivity models and compare their results against real field data. Also, we study different positions and orientations of the source with respect to the casing location and their effects on the EM responses. Furthermore, we consider different casing lengths and diameters to investigate their impact on the EM field. Finally, Section V provides summary remarks and conclusions.

\section{THEORY}

\section{A. Forward modeling}

We consider an Earth model and a 3-D land CSEM configuration whose coordinate system is right-handed with the $z$ axis pointing downwards. The electric dipole transmitter and the receivers measuring EM responses are assumed to be on the Earth's surface and on the $x-y$ plane at $z=0$. As in our previous studies [8], [9], we consider the frequency-domain Maxwell's equations in a diffusive form, hence neglecting dis- placement currents. By assuming a time-harmonic dependence $e^{-i \omega t}$, these equations can be expressed as

$$
\begin{gathered}
\nabla \times \mathbf{E}=i \omega \mu_{0} \mathbf{H}, \\
\nabla \times \mathbf{H}=\mathbf{J}_{\mathrm{s}}+\sigma \mathbf{E},
\end{gathered}
$$

where $\mathbf{E}$ is the electric field, $\mathbf{H}$ is the magnetic field, $\omega$ is the angular frequency, $\mu_{0}$ is the free-space magnetic permeability, $\mathbf{J}_{\mathrm{s}}$ is the distribution of source current, $\sigma \mathbf{E}$ is the induced current in the conductive Earth and

$$
\sigma=\left(\begin{array}{ccc}
\sigma_{x} & 0 & 0 \\
0 & \sigma_{y} & 0 \\
0 & 0 & \sigma_{z}
\end{array}\right)
$$

is the electric conductivity tensor. In eq. (3), $\sigma_{x}, \sigma_{y}$ and $\sigma_{z}$ are known as principal conductivities. Although our formulation works for a general vertical transverse anisotropy case $\left(\sigma_{x}=\right.$ $\sigma_{y} \neq \sigma_{z}$ ), only isotropic cases are considered in this work.

We formulate eq. (1) and eq. (2) in terms of total field. By substituting eq. (1) into eq. (2), we obtain

$$
\nabla \times \nabla \times \mathbf{E}-i \omega \mu_{0} \sigma \mathbf{E}=i \omega \mu_{0} \mathbf{J}_{\mathrm{s}},
$$

which is also known as the curl-curl formulation of the problem in terms of the total field. In our modeling routine, eq. 4 is subject to a homogeneous Dirichlet boundary condition $\mathbf{E}=0$ on the domain boundary.

This approach can avoid numerical errors that arise when the source is located within a region of anomalous properties (e.g., models with high conductivity contrasts or with bathymetry/topography variations) [9], [44]. On the other hand, one disadvantage is that a slightly larger computational domain is required in order to discretize the source properly and avoid reflections from the artificial domain boundaries. To deal with these problems, we have designed adapted meshes.

\section{B. Code details}

We have used a parallel Python code-named PETGEM to compute the EM responses. PETGEM is a code for frequencydomain 3-D CSEM data for marine and land surveys. The High-order Edge Finite Element Method (HEFEM) is used to discretize the governing equations in its diffusive form (in terms of total field as eq. (4)). PETGEM, developed under the BSD-3 license, supports high-order polynomial variants (global $p$-refinement) and unstructured and adapted tetrahedral meshes ( $h$-refinement); arbitrary locations and rotations for receivers and transmitters are allowed; and parallel computations on HPC platforms.

PETGEM has been introduced by [8], where the authors described the numerical formulation and computational details. More concretely, the authors explained the low-order Nédélec Finite Element Method (EFEM) as the numerical scheme for the solution of 3-D marine CSEM and its parallel implementation using Python and third-party libraries. Also, the authors introduced an adaptive-meshing strategy that offers an excellent trade-off between the number of degrees of freedom (dofs) and the predicted EM responses' accuracy. More recently, in [9], a new PETGEM version has been presented. The main improvements consisted of implementing the HEFEM and 

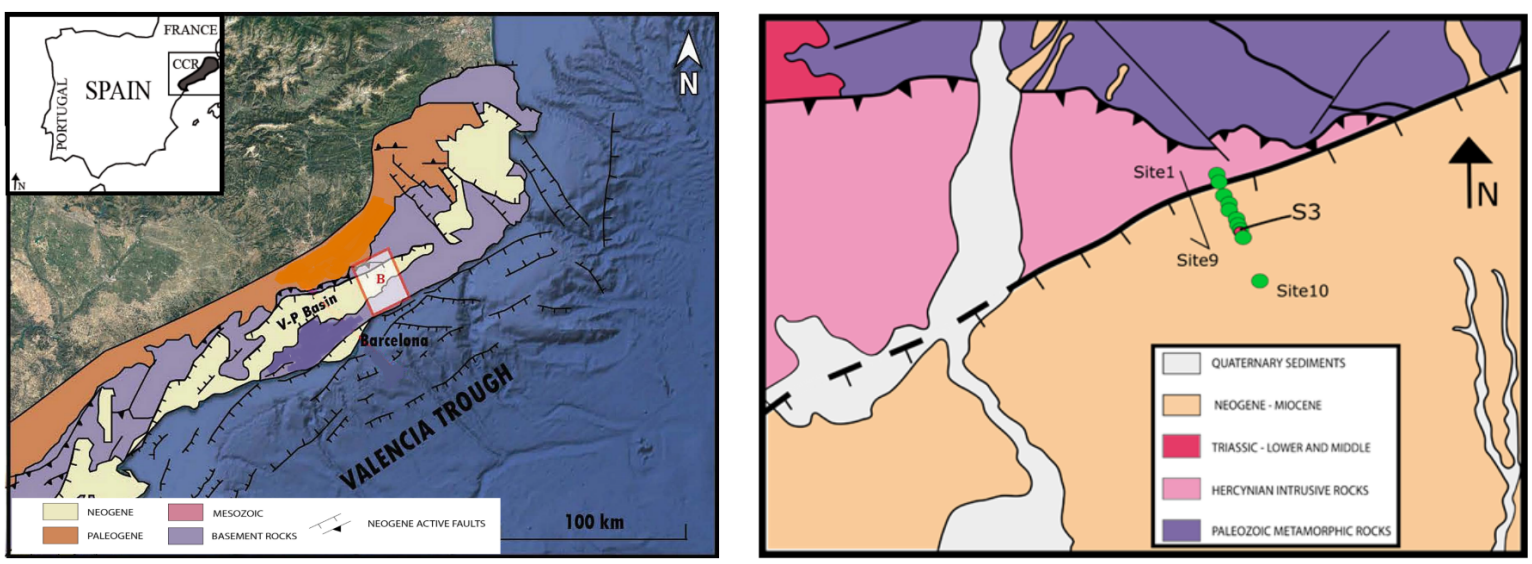

Fig. 1. The Vallès Basin geology. Red rectangle indicates the study area (left panel) and the CSEM receiver/transmitter positions on an NW-SE profile (right panel) marked in green circles. Red circle indicates the metallic casing position used in some configurations (named S3). The profile under consideration is $1300 \mathrm{~m}$ long (from Site 1 to Site 10 in right panel).

the extension of the adaptive-meshing strategy to high-order tetrahedral elements $(p=1,2,3)$. The authors demonstrated that PETGEM offers excellent parallel efficiency for 3-D marine CSEM modeling at both small- and large-scale through a detailed performance scalability analysis. Using HEFEM, and a meshing strategy adapted to the physical parameters, the authors demonstrated a substantial improvement in the numerical precision of simulated data and the generation of meshes capable of capturing the rapid change of the EM fields without increasing the computational cost (e.g., avoiding tetrahedral meshes with a high number of elements). Then, the authors concluded that PETGEM is an efficient and powerful computational tool for the solution of realistic test cases (e.g., regarding geological properties and spatial scales). For more details and proofs, we refer to [8], [9].

It is worth to mention that although PETGEM was initially developed for 3-D marine CSEM problems, the inclusion of other EM configurations requires minimal effort. Several major modules are quite general and independent of the physical method. Thus, they can be reused to develop simulation tools for different types of EM applications (e.g., as in our case, we have implemented and studied the 3-D land CSEM).

\section{ThE VALLÈS: GEOPHYSICAL ACQUISITION SETUP}

Different geophysical surveys have determined the presence of geothermal anomalies in La Garriga-Samalús area in the Vallès Basin (Catalan Coastal Ranges, NE Spain). Nevertheless, the area continues to be poorly understood due to its complex geology that poses challenges for its numerical modeling and characterization. The Vallès basin is a product of different tectonic events, resulting in a fractured system and a fluid circulation regime. Since the Neogene, hydrothermal fluids up to $190^{\circ} \mathrm{C}$ have ascended through the faults and characterized the Vallès fault by hydro-thermal conditions, which remain active until nowadays [45]. Several AMT/MT profiles have imaged the main fault with a reasonable resolution that probably acts as the main conduit for fluids in their way up [46]. The Vallès Basin geology is shown in Fig. 1.
Surface-surface CSEM profiles coincided with the previous AMT/MT profiles and were carried out to assess land CSEM measurements' benefits. Moreover, we have tested several CSEM configurations using existing boreholes with casing located in the area (e.g. for old geothermal research in the eighties) to explore how the source signal and the recorded signal could increase the signal-to-noise ratios. Our study focuses on an NW-SE CSEM profile crossings the Vallès fault of $1300 \mathrm{~m}$ long (see Fig. 1). This profile coincides with a part of a longer MT profile. One of these vintage boreholes (S-3 in Fig. 1) is located on the profile under consideration and could have some effect on the EM data. This borehole is corroded and probably collapsed at some depth, but it does not reach the basement rocks, although the precise actual condition is not well known. No other infrastructures or boreholes are mapped near this profile.

The CSEM acquisition setup consists of a transmitter $\left(T_{x}\right)$ where an EM wave is generated and several receivers $\left(R_{x}\right)$ where the signal is recorded. The transmitter is composed of batteries (10-12 of 12 V), a ZT-30 ZeroTEM (from Zonge) transmitter with a controller (XMT-G from Zonge), a Datalogger (SRU Spider from WorldSensing) that characterizes the signal, a Laptop, a GPS, and two big metallic electrodes separated by $70 \mathrm{~m}$ acting as a big electric dipole source. Each receiver is composed of a short $(10 \mathrm{~m}-20 \mathrm{~m})$ passive electric dipole connected to a data logger. Depending on the ground resistance contact, the electrical intensity was around $2.5 \mathrm{~A}$. The emissions frequencies were $0.125 \mathrm{~Hz}, 0.5 \mathrm{~Hz}, 2 \mathrm{~Hz}$, and $8 \mathrm{~Hz}$. In this profile, different emissions correspond to different positions of $T_{x}$. We used an in-line configuration where $T_{x}$ and $R_{x}$ are parallel.

The emission corresponding to a surface-surface configuration with the $T_{x}$ position at the center (between the receivers 7 and 8 in Fig. 1), is convenient for a joint inversion with the MT sites nearest them. Its joint MT-CSEM 2-D inversion, using MARE2DEM code [47], provides a reference resistivity model for the numerical simulations. The model shown Fig. 2 has an NW-SE orientation crossing the Vallès fault at around 1 $\mathrm{km}$ from the NW limit. It shows two main geoelectrical units. 


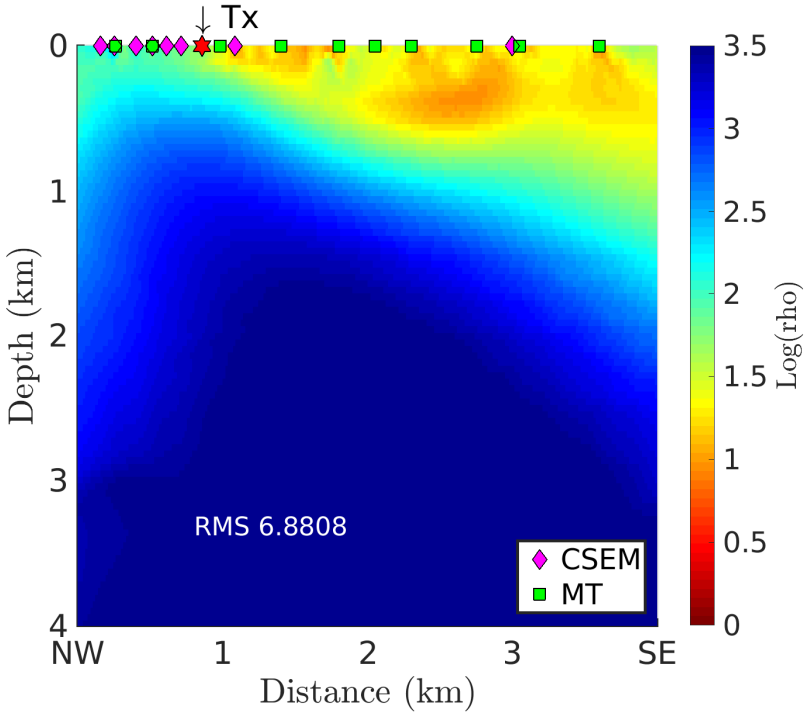

Fig. 2. Joint inversion MT-CSEM surface-surface (using MARE2DEM code) over the profile of interest. Pink diamonds correspond to the CSEM receiver, red star represents the transmitter position. Green squares correspond to the magnetotelluric site stations.

Firstly, the upper unit is a conductive material (resistivities between $3 \Omega \cdot m$ and $50 \Omega \cdot m$ ) that corresponds to the Neogene sediments filling the basin. Secondly, the resistive basement correlated with granite rocks having a strong alteration in its upper limits, where the reservoir is developed. The model imaged a SE dipping topography of the basement that can be interpreted as the sequence of normal faults.

\section{Simulations}

To perform the numerical simulations, we have generated a simplified 3-D resistivity model from its 2-D counterpart described in Fig. 2. The resulting 3-D resistivity model is depicted in Fig. 3, which is composed of three materials: a $4 \mathrm{~km}$ thick air layer $\left(10^{8} \Omega \cdot \mathrm{m}\right)$, resistive basement $\left(\rho_{1}\right)$ including topography, and the conductive sediments $\left(\rho_{2}\right)$. Over imposed to this reference model, we include a vertical cylinder $\left(\rho_{3}\right)$ embedded in the sediments to represent the metal well casing in the area. It is centered at $x=1719 \mathrm{~m}, y=2000 \mathrm{~m}$, and $z=-100 \mathrm{~m}$. The cylinder length is $200 \mathrm{~m}$. We have studied different resistivity values for $\rho_{1}, \rho_{2}$, and $\rho_{3}$ (see details in Table I). To avoid huge computational resources when the actual casing parameters are modeled, our cylinder has a diameter of $5 \mathrm{~m}$ and resistivity values of $10^{-3} \Omega \cdot \mathrm{m}$ and $5 \cdot 10^{-3} \Omega \cdot \mathrm{m}$. Therefore, $\rho_{3}$ preserves the equivalent casing conductance [48] to the estimated one of the old boreholes (order of $10^{4} \mathrm{~S} \cdot \mathrm{m}$ ).

The computational domain is defined by $[0,6] \times[-1,5] \times$ $[-5,5] \mathrm{km}$. To improve the accuracy of EM responses, we have applied a mesh refinement close to source and receiver locations based on the semi-automatic refinement strategy proposed by [9]. Further, we applied a power-law stretching to control and adapt the number of points near the casing and account for extreme element aspect ratios [32], [33], [37]. The resulting adapted and unstructured tetrahedral mesh consists of 840385 elements and 138573 nodes. We used polynomial basis of second-order $(p=2)$ for all experiments, which produces a linear equation system with 5344746 dofs. A 2$\mathrm{D}$ view of the computational domain under consideration is depicted in Fig. 3.

We have used a suite of three fundamental dipole sources at $2 \mathrm{~Hz}$ with a moment of $1 \mathrm{~A} \cdot \mathrm{m}$. The first one is a horizontal electric dipole (HED) located at $x=1209 \mathrm{~m}, y=2000 \mathrm{~m}$, and $z=-2 \mathrm{~m}\left(T_{x}\right.$ in Fig. 3). The second source is a vertical electric dipole (VED) located at $x=1719 \mathrm{~m}, y=2000 \mathrm{~m}$, and $z=-2 \mathrm{~m}\left(T_{z 1}\right.$ in Fig. 3). Finally, the third source is a deep vertical electric dipole (dVED) situated at $x=1719 \mathrm{~m}, y=$ $2000 \mathrm{~m}$, and $z=-204 \mathrm{~m}$ ( $T_{z 2}$ in Fig. 3). To investigate the EM responses, we have designed a grid of receiver positions with 39 in-line profiles pointing to NW-SE. Each profile has 33 receivers and a length of $2438 \mathrm{~m}$. The receivers' grid is denser in the vicinity of the metallic casing, which allow us to better analyze the EM field pattern in that area.

We simulate different setups that allow us a progression from the most simple case to the most complex, including the metallic casing effect. Table I shows the experiment details (source type, case label, resistivity values). Table I also includes the appropriate reference to figures where the simulation results are presented. In the next sections, we will discuss the most relevant cases. All simulations have been carried out on Marenostrum IV supercomputer, whose current peak performance is 13.7 Petaflops. It is a Lenovo system composed of 165888 processor cores at $2.10 \mathrm{GHz}$ grouped into 3456 computing nodes, $390 \mathrm{~TB}$ of main memory, and 14 PB of GPFS disk storage. Each computing node has two sockets with 24 cores with a total of 48 cores per node.

\section{A. Validation against analytic solution}

As the first example, we study the numerical error for a homogeneous medium comparing with DIPOLE1D code [49]. For this experiment, we considered the most straightforward cases to control numerical accuracy, namely $T_{x} \beta \beta \beta$ and $T_{z 1} \beta \beta \beta$ (see Table I). It is worth mentioning that the numerical results derived below remain valid for other simple case comparisons such as $T_{x} \alpha \beta \beta$ against $T_{z 1} \alpha \beta \beta$ or $T_{z 2} \alpha \beta \beta$. Still, to preserve brevity, we focus on homogeneous cases.

For each test case, Fig. 4 shows the electric field amplitude $\left|\mathbf{E}_{x}\right|$ along the first receiver line $(z=-2)$. We consider an $L^{2}$ norm to quantify the average misfits of the numerical solution $\mathcal{Q}_{h}$ with respect to the analytical solution $\mathcal{Q}_{e}$. The $L^{2}$-norm can be stated as

$$
\epsilon_{L^{2}}=\left\|\mathcal{Q}_{h}-\mathcal{Q}_{e}\right\|_{L^{2}(\Omega)}=\left(\int_{\Omega}\left|\mathcal{Q}_{h}-\mathcal{Q}_{e}\right|^{2} d V\right)^{1 / 2} .
$$

This norm involves the use of FE basis functions to interpolate the discrete solution to any point at the computational domain $\Omega$ [9]. For both amplitude quantities, the results are almost identical with respect to the analytical solution. More concretely, for case $T_{x} \beta \beta \beta$, the misfit average is 3.48 percent except near the source (less than $80 \mathrm{~m}$ ). On the other hand, we obtained 1.18 percent of misfit average for case $T_{z 1} \beta \beta \beta$, 


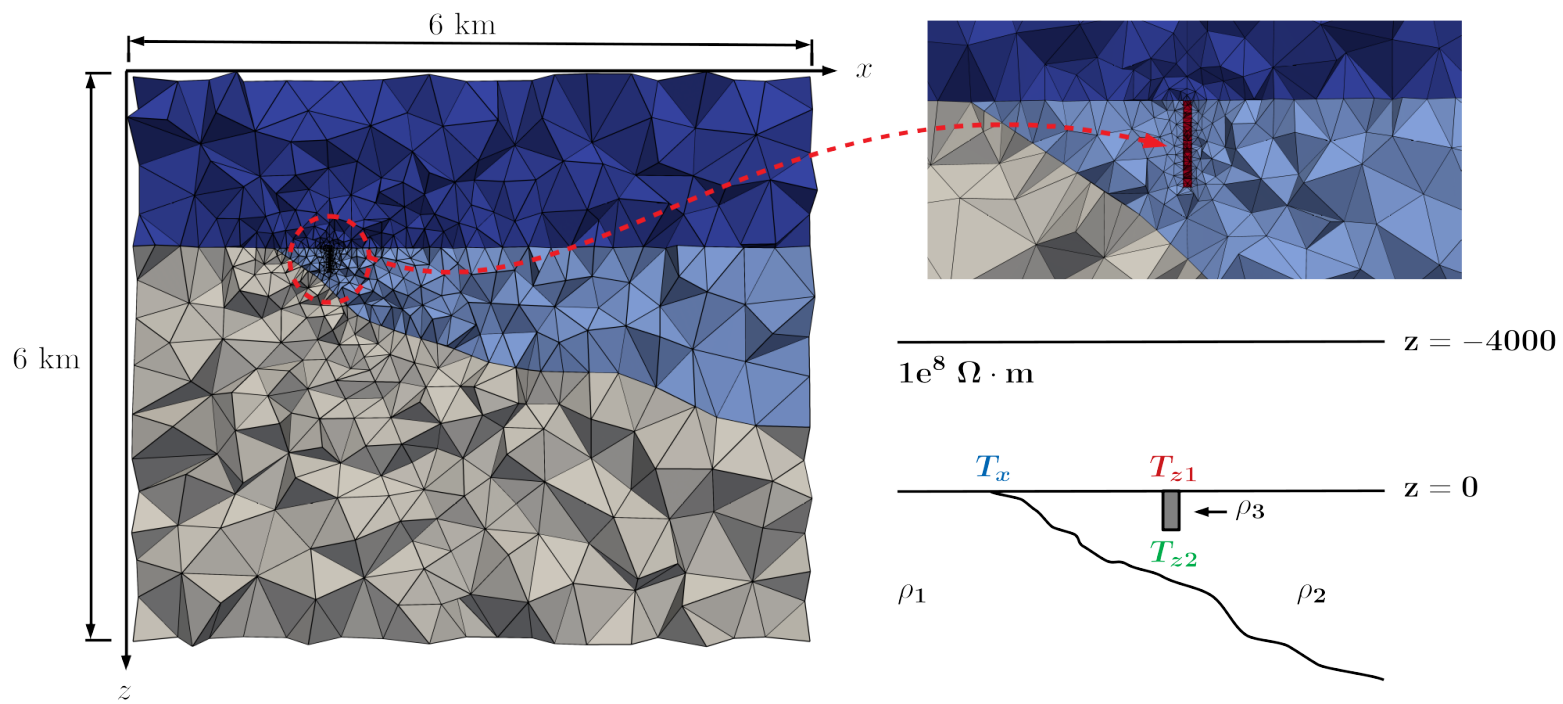

Fig. 3. 3-D CSEM model under consideration with its resulting computational unstructured and $h$-adapted mesh. The mesh view corresponds to $y=2000 \mathrm{~m}$ with zoom-in close to casing location. The color scale is illustrative; therefore it does not represent the actual model parameters.

TABLE I

SUMMARY OF THE MOST RELEVANT SCENARIOS STUDIED. SOURCE TYPE, CASE LABEL, RESISTIVITY VALUES (EXPRESSED IN $\Omega \cdot M$ ), AND ITS CORRESPONDING FIGURE ARE INCLUDED. THE TEST CASE NAMING CONVENTION IS SOURCE $\rho_{1} \rho_{2} \rho_{3}$

\begin{tabular}{|c|c|c|c|c|c|}
\hline $\begin{array}{l}\text { Source } \\
\text { type }\end{array}$ & $\begin{array}{l}\text { Case } \\
\text { label }\end{array}$ & $\begin{array}{c}\rho_{1} \\
\text { (Basement) }\end{array}$ & $\begin{array}{c}\rho_{2} \\
\text { (Sediments) }\end{array}$ & $\begin{array}{c}\rho_{3} \\
\text { (Casing) }\end{array}$ & Figure \\
\hline \multirow{6}{*}{ HED } & $T_{x} \beta \beta \beta$ & $\beta$ & $\beta$ & $\beta$ & $4,5,7$ \\
\hline & $T_{x} \alpha \beta \beta$ & $\alpha$ & $\beta$ & $\beta$ & 9 \\
\hline & $T_{x} \alpha \beta \gamma$ & $\alpha$ & $\beta$ & $\gamma$ & 7 \\
\hline & $T_{x} \alpha \beta \delta$ & $\alpha$ & $\beta$ & $\delta$ & $7,8,9,10,11$ \\
\hline & $T_{x} \beta \beta \gamma$ & $\beta$ & $\beta$ & $\gamma$ & 7 \\
\hline & $T_{x} \beta \beta \delta$ & $\beta$ & $\beta$ & $\delta$ & 7 \\
\hline \multirow{6}{*}{ VED } & $T_{z 1} \beta \beta \beta$ & $\beta$ & $\beta$ & $\beta$ & 4,5 \\
\hline & $T_{z 1} \alpha \beta \beta$ & $\alpha$ & $\beta$ & $\beta$ & \\
\hline & $T_{z 1} \alpha \beta \gamma$ & $\alpha$ & $\beta$ & $\gamma$ & 6 \\
\hline & $T_{z 1} \alpha \beta \delta$ & $\alpha$ & $\beta$ & $\delta$ & 6 \\
\hline & $T_{z 1} \beta \beta \gamma$ & $\beta$ & $\beta$ & $\gamma$ & 5,6 \\
\hline & $T_{z 1} \beta \beta \delta$ & $\beta$ & $\beta$ & $\delta$ & 5 \\
\hline \multirow{6}{*}{ dVED } & $T_{z 2} \alpha \beta \beta$ & $\alpha$ & $\beta$ & $\beta$ & \\
\hline & $T_{z 2} \alpha \beta \gamma$ & $\alpha$ & $\beta$ & $\gamma$ & 6 \\
\hline & $T_{z 2} \alpha \beta \delta$ & $\alpha$ & $\beta$ & $\delta$ & 6 \\
\hline & $T_{z 2} \beta \beta \gamma$ & $\beta$ & $\beta$ & $\gamma$ & 5,6 \\
\hline & $T_{z 2} \beta \beta \delta$ & $\beta$ & $\beta$ & $\delta$ & 5 \\
\hline & $\alpha=$ & $000 \quad \beta=20$ & $\gamma=0.005$ & $\delta=0.001$ & \\
\hline
\end{tabular}

excluding points close to source position (less than $20 \mathrm{~m}$ ). Moreover, the reciprocity principle has also been tested with a misfit of 0.01 percent.

Note that both results are subject to mesh quality. The characteristic mesh spacing near $T_{x}$ is about $20 \mathrm{~m}$, while for $T_{z 1}$ vicinity, mesh element size is around $0.8 \mathrm{~m}$. Consequently, element size close to $T_{z 1}$ (casing region) is about 25 times smaller than the element size near to $T_{x}$ location. The latter results in more capability to capture the rapid change of the EM field. However, despite of the considerable differences in the element size ratio, misfits are all very accurate. To confirm these results, we perform a mesh convergence test.
The numerical solutions have been computed on a set of globally $h p$-refined meshes, starting from an adapted mesh for $p=2$ with 840385 elements (level 1) and ending in a fine mesh with 2110258 elements (level 3). The mesh hierarchies are shown in Table II. The convergence results, run-time (in minutes), and memory (in $\mathrm{Gb}$ ) are also depicted in Table II. We consider run-time as the elapsed real-world time from start to end of solving the sparse linear system. Memory refers to the maximum peak of memory consumption at any point of solving phase. For all mesh levels, piece-wise $p=2$ approximation produces the best trade-off between numerical accuracy and computing effort because the reference mesh has 


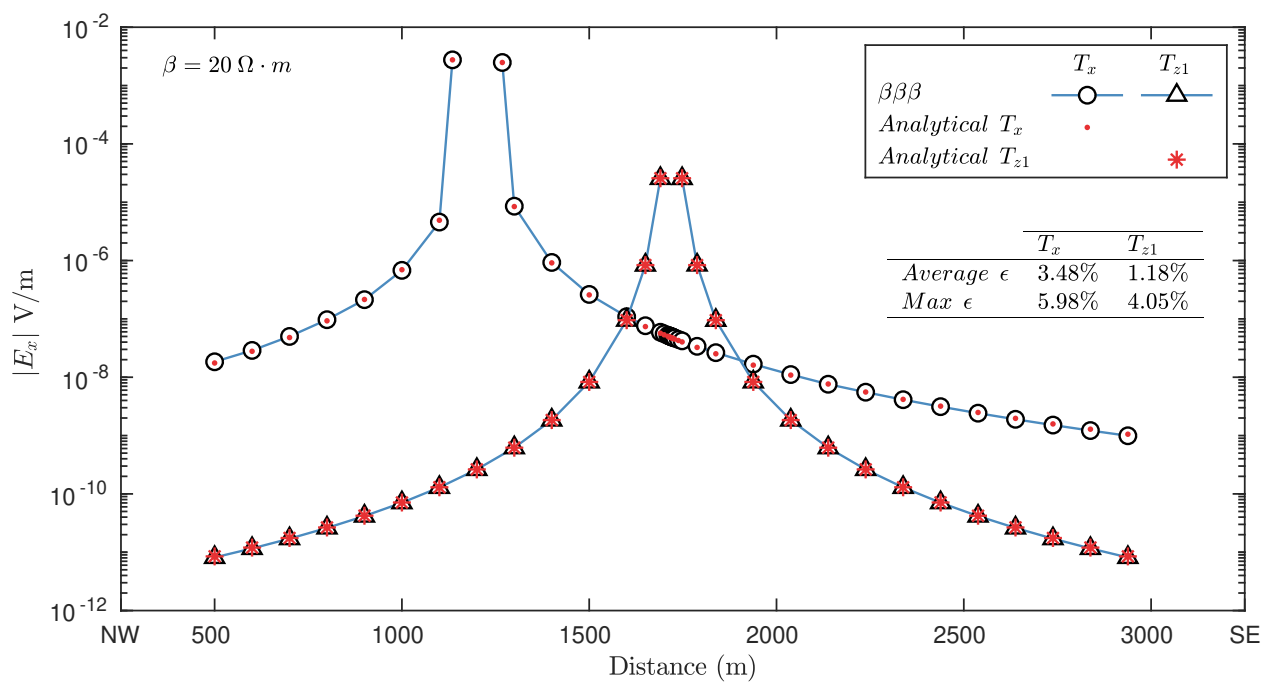

Fig. 4. Comparison of in-line electric field amplitudes $\left|\mathbf{E}_{x}\right|$ obtained with PETGEM against analytical solutions. Horizontal source $T_{x}$ and vertical source $T_{z 1}$ are plotted against its reference for the homogeneous model $\beta \beta \beta$.

TABLE II

Mesh STATISTICS. NUMBER OF ElEMENTS, DOFS, Misfit AVERAGE $\epsilon$, RUN-TIME, AND MEMORY CONSUMPTION FOR TEST CASES $T_{x} \beta \beta \beta$ AND $T_{z 1} \beta \beta \beta$. THREE DIFFERENT FE BASIS POLYNOMIAL ORDERS HAVE BEEN USED $(p=1,2,3)$.

\begin{tabular}{cccccc}
\hline \multirow{2}{*}{$p$} & \multicolumn{5}{c}{ Mesh level 1 (840 385 elements) } \\
\cline { 2 - 5 } & dofs & $\epsilon\left(T_{x} \beta \beta \beta\right)$ & $\epsilon\left(T_{z 1} \beta \beta \beta\right)$ & Run-time (min) & Memory $(\mathrm{Gb})$ \\
\hline 1 & 985280 & 9.84 & 9.12 & 2.37 & 42.32 \\
2 & 5344746 & 3.48 & 1.18 & 10.36 & 250.12 \\
3 & 15599553 & 2.65 & 0.95 & 38.14 & 497.85 \\
\hline
\end{tabular}

\begin{tabular}{cccccc}
\multicolumn{5}{c}{ Mesh level 2 (1 563 137 elements) } \\
\hline 1 & 1832680 & 6.19 & 5.79 & 4.78 & 78.12 \\
2 & 8762067 & 2.25 & 0.90 & 13.45 & 294.36 \\
3 & 23132637 & 1.85 & 0.68 & 65.36 & 538.68 \\
\hline
\end{tabular}

\begin{tabular}{cccccc}
\multicolumn{5}{c}{ Mesh level 3 (2 110 258 elements) } \\
\hline 1 & 2329741 & 4.05 & 3.93 & 6.89 & 97.36 \\
2 & 10134512 & 1.15 & 0.55 & 16.14 & 320.47 \\
3 & 28755081 & 0.38 & 0.15 & 80.66 & 673.49 \\
\hline
\end{tabular}

been adapted for this piece-wise order. Consequently, although the piece-wise $p=3$ is more accurate than piece-wise $p=2$, its memory consumption and run-time are too demanding with respect to numerical precision improvement. The piece-wise $p=1$ is the least accurate and most economical in memory and run-time consumption. Therefore, we conclude that the adapted mesh strategy can provide acceptable error levels while avoiding excessive refinement in the whole computational domain. However, the best performance in terms of speed and numerical precision depends on the input model and the FE basis polynomial degrees, which guides the mesh adaptation process [9].

We point out that, in these examples, the electric field amplitude has a stronger decrease for the vertical source case $\left(T_{z 1}\right)$ than for the horizontal case $\left(T_{x}\right)$. Based on these results, we conclude that although $T_{x}$ is the typical configuration for a realistic case, the use of a vertical source as $T_{z 1}$ is also feasible in the presence of a borehole. This effect is analyzed in detail below.

\section{B. Casing effect with a vertical source}

As a second example, we investigate the metallic casing effect on EM responses using vertical sources. This experiment is relevant because electric fields in the use of vertical sources decay more rapidly in comparison to those in the use of horizontal sources (see Fig. 4). We compare the electric field pattern produced by vertical sources located at the top $\left(T_{z 1}\right)$ and the bottom $\left(T_{z 2}\right)$ of the casing. Fig. 5 depicts how the presence of a metallic casing alters the electric field amplitude pattern. Comparisons of cases with $T_{z 1}$ at the surface (cases $T_{z 1} \beta \beta \beta, T_{z 1} \beta \beta \gamma$, and $T_{z 1} \beta \beta \delta$ ) show that the presence of a metallic casing produces a stronger decrease in the electric field with respect to source offset. However, if the source is located at the bottom of the metallic casing (cases $T_{z 2} \beta \beta \gamma$ and $T_{z 2} \beta \beta \delta$ ), the electric field is amplified about 4 orders of magnitude. Consequently, the electric field amplitude produced by 


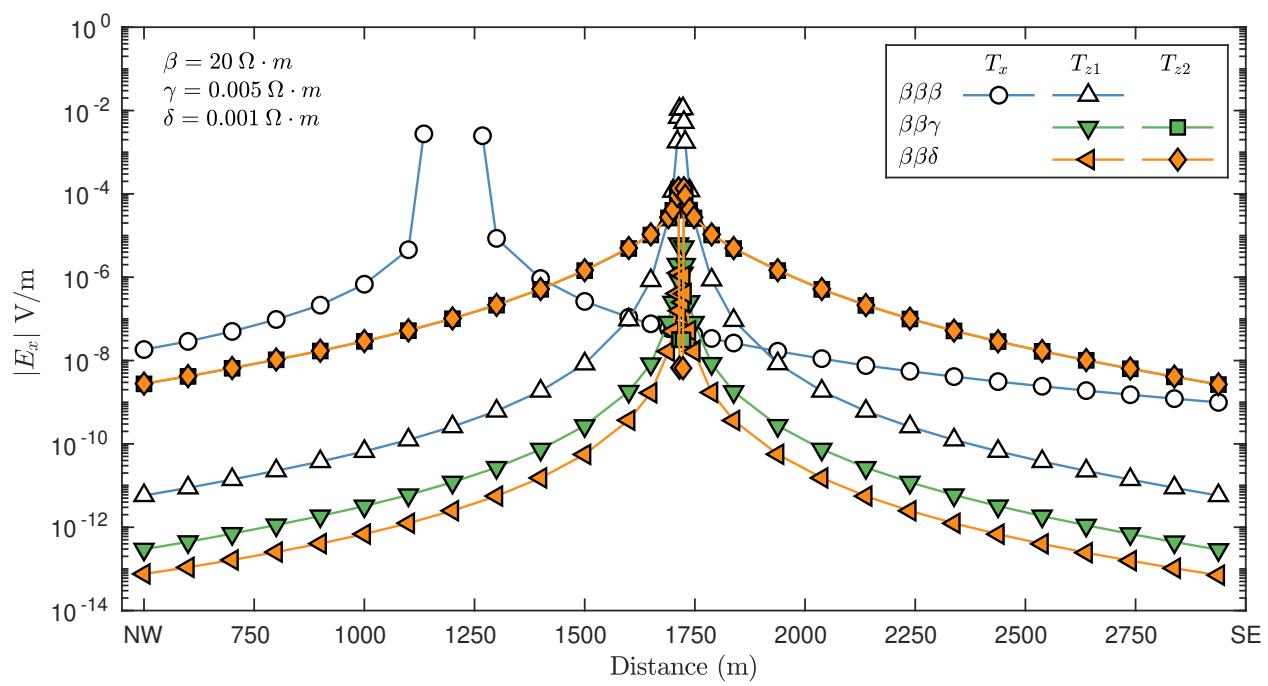

Fig. 5. Metallic casing effect on the electric field amplitude pattern $\left|\mathbf{E}_{x}\right|$ generated by vertical sources $\left(T_{z 1}\right.$ and $\left.T_{z 2}\right)$ in an homogeneous medium (Basement $\left(\rho_{1}\right)=$ Sediments $\left.\left(\rho_{2}\right)\right)$. Note that $\left|\mathbf{E}_{x}\right|$ for case $T_{x} \beta \beta \beta$ is obtained using a horizontal source $T_{x}$.

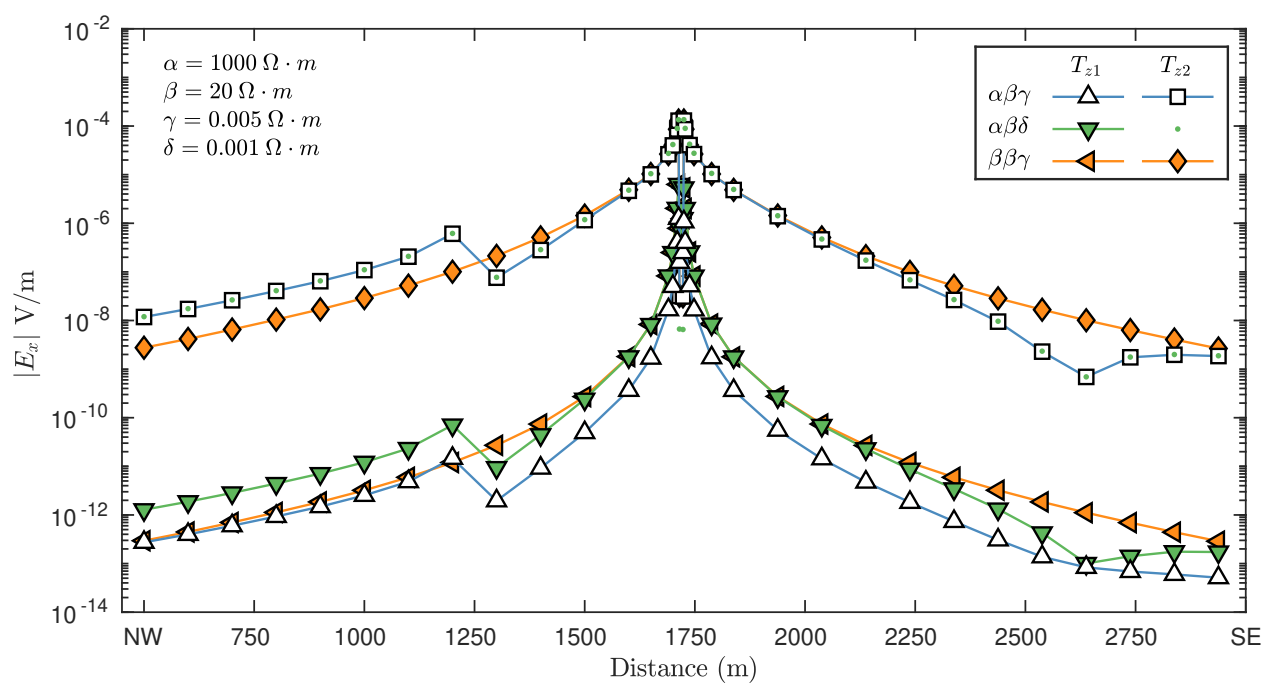

Fig. 6. Metallic casing effect on the electric field amplitude pattern $\left|\mathbf{E}_{x}\right|$ generated by vertical sources $\left(T_{z 1}\right.$ and $\left.T_{z 2}\right)$ in a non-homogeneous medium (Basement $\left(\rho_{1}\right) \neq$ Sediments $\left.\left(\rho_{2}\right)\right)$.

vertical source $T_{z 2}$ reaches the amplitude level generated by horizontal source $T_{x}$. Moreover, in our experiments, the effect of casing conductivity is not very important (compare electric field amplitudes for cases $T_{z 1} \beta \beta \gamma$ and $T_{z 1} \beta \beta \delta$ in Fig. 5), and negligible when the source is located below the metallic casing (case $T_{z 2} \beta \beta \gamma$ versus case $T_{z 2} \beta \beta \delta$ in Fig. 5).

Once the effect in a homogeneous setup has been investigated, we analyze EM responses for the same metallic casing and source configurations but in a non-homogeneous medium, including a non-horizontal resistive basement with topography. For each source type $\left(T_{z 1}\right.$ and $\left.T_{z 2}\right)$, the resistivity models under consideration are $\alpha \beta \gamma, \alpha \beta \delta$, and $\beta \beta \gamma$. The amplitude $\left|\mathbf{E}_{x}\right|$ of the electric field responses are shown in Fig. 6. It can be seen that the electric field pattern is the same as for the homogeneous medium (see Fig. 5), but the presence of characteristics "peaks" (at around $x=1200 \mathrm{~m}$ and $x=2700 \mathrm{~m}$ ) reveals the heterogeneity of the medium in particular the presence of a resistor at NW. This resistivity mapping is as expected and has been widely analyzed in previous CSEM studies [4], [6], [8], [9], [44], [49]. In our simulations, the casing is represented as a solid material. Therefore, the EM fields along the metal well casing decay exponentially until its amplitude reaches zero at its bottom. This amplitude decay behavior is consistent with other simulation results where the steel casing effect on EM responses is also studied [16], [37], [50], [51].

\section{Effect of a casing distant to a horizontal source}

As a third example, we study the EM responses when the metallic casing is distant from a horizontal source $\left(T_{x}\right)$. Homogeneous and non-homogeneous media have been used to analyze this effect. Previous studies investigated EM field pattern produced by VED in different applications such as hydrocarbon exploration [50], reservoir monitoring [52], and 


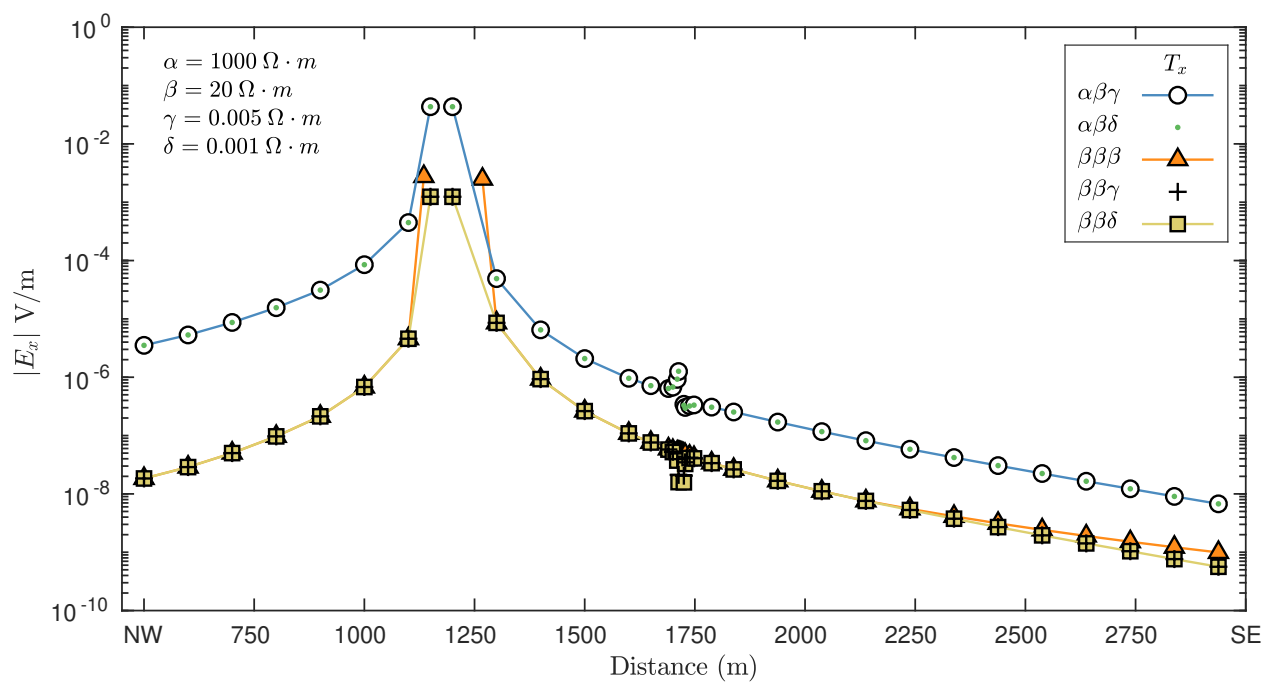

Fig. 7. Electric field amplitude $\left|\mathbf{E}_{x}\right|$ in the presence of a metallic casing distant to horizontal source $T_{x}$. Homogeneous and non-homogeneous medium are compared.

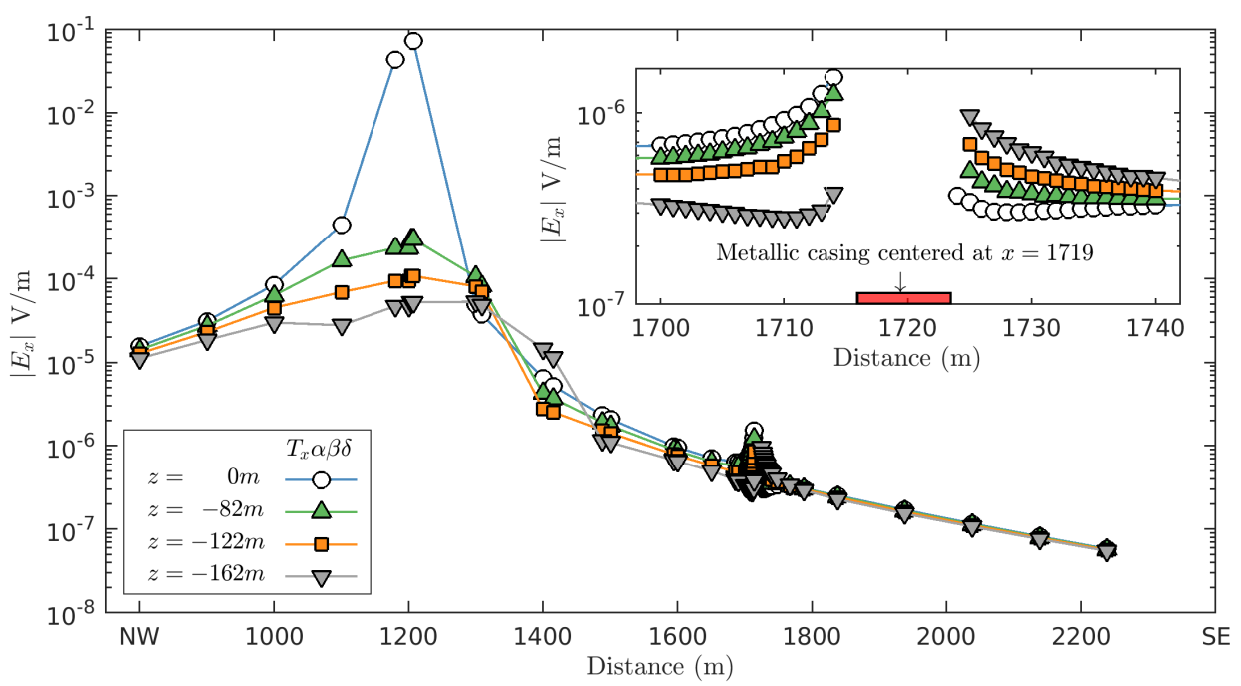

Fig. 8. Electric field behavior closer to metallic casing vicinity for case $T_{x} \alpha \beta \delta$. Electric field amplitude $\left|\mathbf{E}_{x}\right|$ is plotted for different receiver depths in the $z$-axis. The spatial location of the metallic casing is also included.

$\mathrm{CO}_{2}$ storage [16], [37]. Therefore, one of our main paper contributions consists of analyzing EM responses generated by HED in the presence of steel-cased wells and its application in a geothermal exploration context.

For this experiment, we used different resistivity cases. The first one consists of a conductive medium (sediments) with different resistive basement (cases $T_{x} \alpha \beta \gamma$ and $T_{x} \alpha \beta \delta$ ). The second one corresponds to a model with a metallic casing embedded in a homogeneous medium (cases $T_{x} \beta \beta \gamma$ and $T_{x} \beta \beta \delta$ ). Finally, the third scenario consists of a homogeneous medium with no well casing (case $T_{x} \beta \beta \beta$ ).

The electric field amplitudes $\left|\mathbf{E}_{x}\right|$ for each resistivity case are depicted in Fig. 7. For cases where the casing is present, the electric field amplitudes are only altered near the casing (cases $T_{x} \alpha \beta \gamma, T_{x} \alpha \beta \delta, T_{x} \beta \beta \gamma$, and $T_{x} \beta \beta \delta$ ), which corresponds to a local effect $(x=1620 \mathrm{~m}$ to $x=1740 \mathrm{~m})$. It is also easy to see that the general behavior corresponds to that of the horizontal source $T_{x}$ in a 2-D medium with a resistive zone at the NW part. Also, the individual comparison of cases $T_{x} \alpha \beta \gamma$ and $T_{x} \alpha \beta \delta$ confirms that the impact of different casing conductivity values is negligible. This behaviour is consistent for cases $T_{x} \beta \beta \gamma$ and $T_{x} \beta \beta \delta$.

For case $T_{x} \alpha \beta \delta$, Fig. 8 depicts a detailed view of the electric field behavior closer to the metallic casing vicinity and for deeper receiver positions. For this test, an increase of around 0.5 or 1 order of magnitude is obtained. It is worth to mention that this effect could be used to obtain higher signal-to-noise ratios in the $R_{x}$ near the casing location.

Fig. 9 shows the results for an emission in-situ of the Vallès basin profile. The $T_{x}$ is located at position 2 and the borehole is used as an $R_{x}$ (see right panel of Fig. 1). For this experiment, we connected $R_{x}$ directly to the metallic casing. 


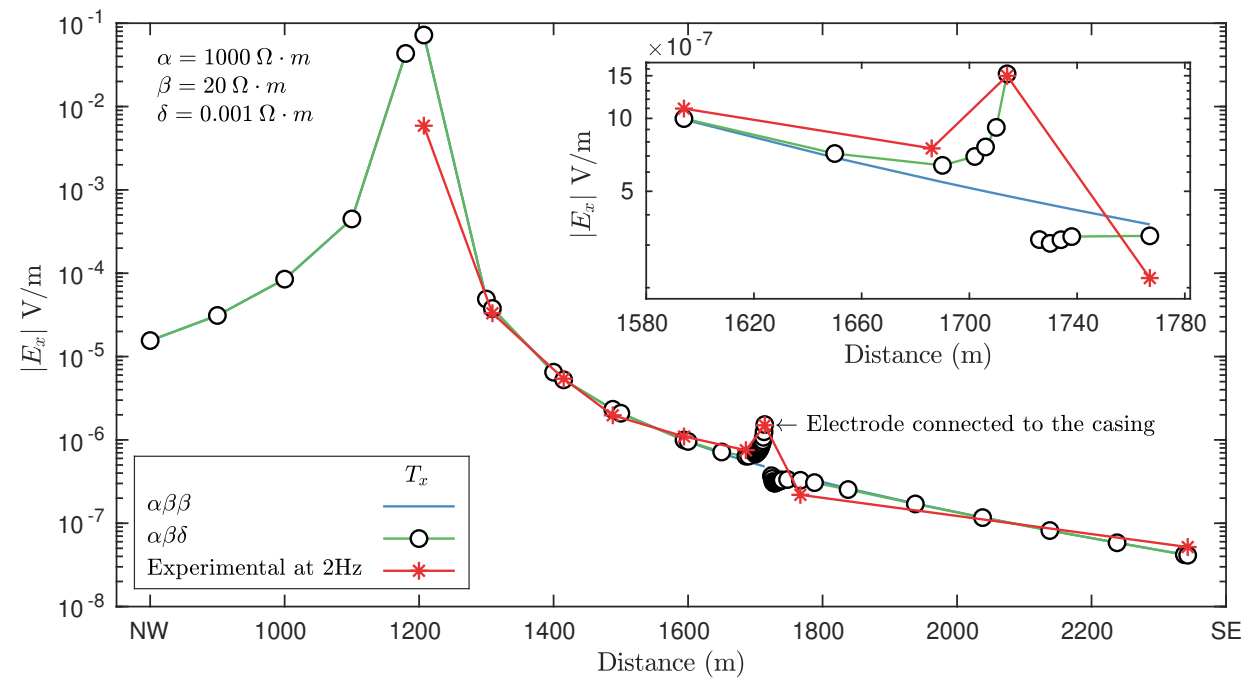

Fig. 9. Experimental data in-situ of the Vallès Basin profile compared with numerical case $T_{x} \alpha \beta \delta$. The experimental data amplitude has been shifted up by a factor of 53.03, obtaining a RMS of 1.382 .

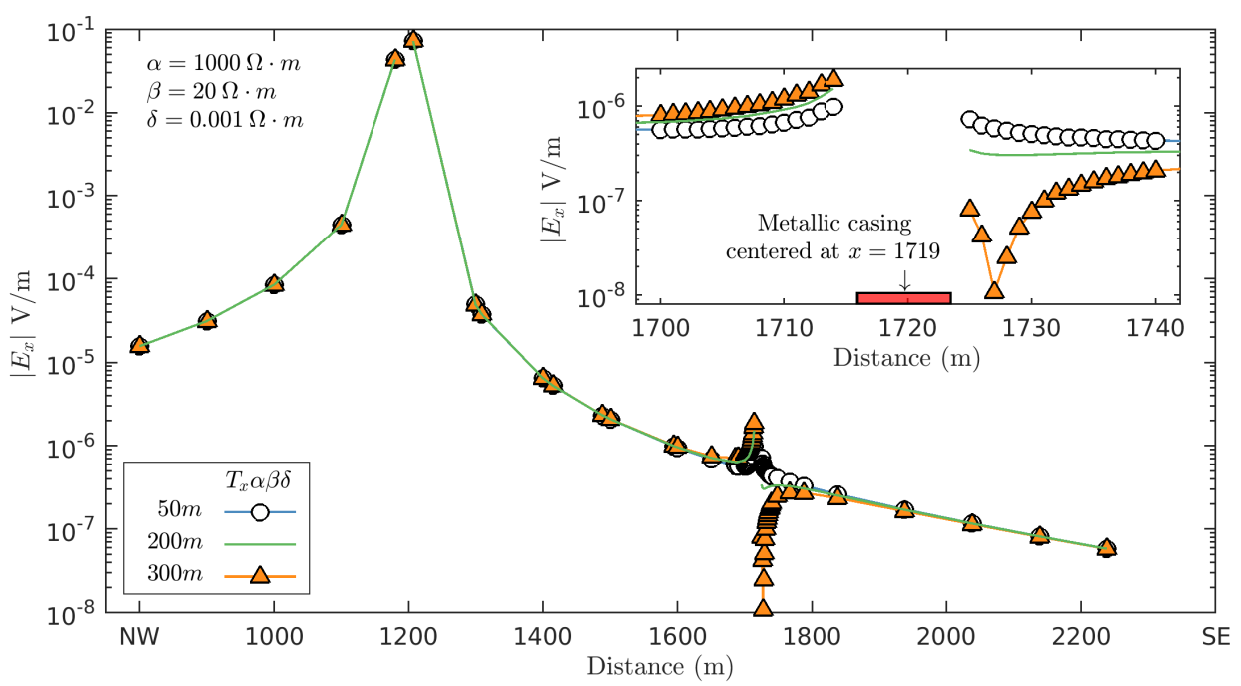

Fig. 10. Effect of different well casing lengths on EM responses for case $T_{x} \alpha \beta \delta$. Three well casing lengths are presented: $50 \mathrm{~m}, 200 \mathrm{~m}, 300 \mathrm{~m}$.

To compare synthetic EM responses against the experimental measurements, the electrical amplitude $\left|\mathbf{E}_{x}\right|$ of cases $T_{x} \alpha \beta \beta$ and $T_{x} \alpha \beta \delta$ are superimposed. To fit experimental amplitudes, they are plotted with a constant downshift factor of 53.03 for all $x$, getting a root mean squared (RMS) of 1.382 . As electric field is mainly proportional to the medium's resistivity, this downshift indicates that the sediments' resistivity is, in average, much lower than the $20 \Omega \cdot \mathrm{m}$ considered in the 3-D model used for our simulations. The electric field amplitudes depicted in Fig. 9 confirm the effect of the metallic casing presence, notably close to its vicinity. More concretely, the casing presence alters the electric field pattern by approximately 1 order of magnitude compared to the model with no well casing (case $T_{x} \alpha \beta \beta$ ). Also, a close inspection of Fig. 9 shows that the field amplitude is modified by a distance of around $120 \mathrm{~m}$ (from $x=1620 \mathrm{~m}$ to $x=1740 \mathrm{~m}$ ).

\section{Effect of casing length and diameter}

Finally, the fourth test focuses on studying the impact of well casing length and diameter on the EM responses. To simulate these cases, we regenerate the unstructured tetrahedral mesh by considering the new spatial dimensions of the casing.

As first part of this experiment, we compute the EM fields for two additional casing lengths, namely $50 \mathrm{~m}$ and $300 \mathrm{~m}$ (increase/decrease casing length, respectively). Fig. 10 depicts the electric field amplitude $\left|\mathbf{E}_{x}\right|$ for each casing length. It is easy to see that the the pattern of the electric field is the same for receiver positions distant to the casing location. However, for positions close to the metal structure (from $x=1600 \mathrm{~m}$ to $x=1838 \mathrm{~m}$ ), electric field amplitudes show a difference of around 1 order of magnitude, which suggests that different well casing lengths and their local impact on EM pattern are not negligible (at least about $200 \mathrm{~m}$ away from the casing 


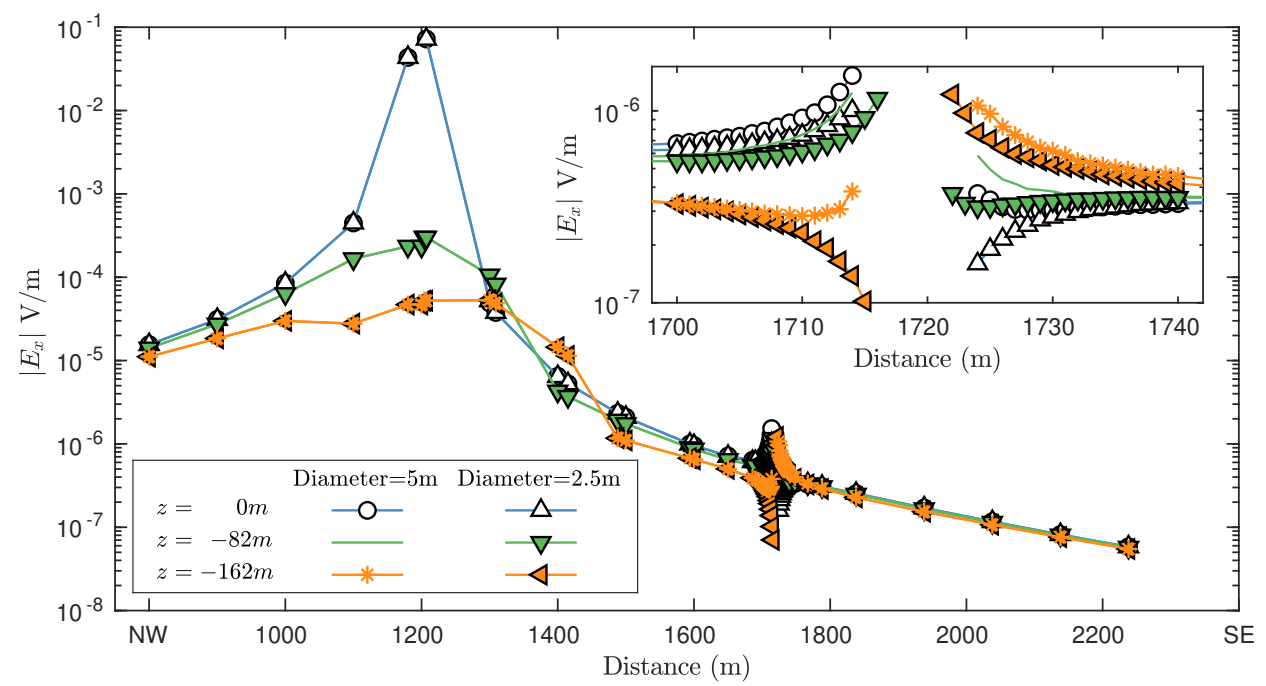

Fig. 11. Effect of different casing diameters on the electric field amplitude $\left|\mathbf{E}_{x}\right|$ for case $T_{x} \alpha \beta \delta$. Two casing diameters are studied: $5 \mathrm{~m}$ and $2.5 \mathrm{~m}$.

location). It is also worth to mention that for the case with the most extensive casing length $(300 \mathrm{~m})$, the electric amplitude reveals a significant alteration around $x=1727 \mathrm{~m}$. This variation is because the bottom of the casing is very close to the basement surface $(\approx 30 \mathrm{~m})$, causing additional reflection on EM fields in that region.

As the second part of this experiment, we consider a $200 \mathrm{~m}$ casing (initial configuration) and reduce its diameter to $2.5 \mathrm{~m}$. Then, for this casing setup we compute the EM responses. Fig. 11 shows the electric field amplitude $\left|\mathbf{E}_{x}\right|$ for each casing diameter $(5 \mathrm{~m}$ versus $2.5 \mathrm{~m}$ ). Similar to casing length, the radius has a negligible impact on the general EM field pattern. It only alters the EM responses in the casing vicinity by 1 order of magnitude, confirming its local effect.

\section{CONCLUSIONS}

We have presented a set of 3-D land CSEM simulations in the presence of metallic well casing for the Vallès Basin. The importance of these experiments is based on two main reasons. Firstly, previous studies have described the region's enormous potential as a resource to produce heat due to geothermal anomalies present there [43]. Secondly, it is well known that metallic infrastructure alters EM responses of deep bodies or targets, which naturally ends up in incomplete or erroneous interpretations. However, studying the effect of metallic casing on EM responses for realistic configurations is a non-trivial task from a numerical modeling perspective (e.g., highly conductivity contrasts, large-scale variations, complex geometries including topography, among others). Fortunately, although numerically challenging, a realistic 3-D land CSEM modeling in the presence of metallic casing is feasible given the continuous improvement of numerical approaches and computing power, as shown in this paper.

We study the metallic casing effect by considering different CSEM configurations, starting with validations for simple models. From there, we increase the complexity to bring them closer to realistic cases. We have generated a 3-D resistivity model from an MT-CSEM 2-D inversion to perform the
PETGEM simulations. Numerical results confirm that metallic casing presence strongly influences EM responses, but its effect is limited to the close vicinity of the steel-cased well. More concretely, in our experiments, the electric field pattern decay depends on the source position (top or bottom of the casing), source direction (horizontal or vertical), and depth of receiver where EM responses is measured. Building upon the configuration, the electric field behavior closer to the metallic casing is altered in different ranges: of around 4 orders of magnitude (e.g., a vertical source in non-homogeneous medium depicted in Fig. 5) or an increase between 0.5 and 1 order of magnitude (e.g., horizontal source distant to the casing in non-homogeneous medium depicted in Fig. 7). The metallic casing produced an effect that makes surface measurements more sensitive to resistivity variations near the metallic structure (similar effect to those obtained by [37], [50], [53]). Simulation results also showed a reduced impact on the electric fields from the variation of casing's conductivity (reduced impact when the source is placed above the casing and negligible when it is located below the casing, see Fig. 6). Furthermore, synthetic EM responses confirm the impact of different well casing lengths and radius on the electric field behavior. However, this distortion is reduced and only effective near to casing (local and around $100 \mathrm{~m}$ ). Consequently, its impact depends on where the steel casing is positioned with respect to the transmitter. Still, the impact on EM measurements should be taken into account to avoid misunderstanding interpretations. Moreover, electric field amplitude along the steel-cased well decays exponentially until it reaches zero at the bottom of the metallic structure. From these experiments, we conclude that the metallic casing effect, depending on the input model (e.g., conductivity contrasts, frequency, geometry, mesh quality), could be used to improve the signal-to-noise ratios on receivers $R_{x}$ close to vicinity of the steel-cased well. This conclusion is consistent to others obtained in previous applications [16], [37]. Hence, the numerical results presented here, are relevant for applications such as $\mathrm{CO}_{2}$ storage and geothermal exploration, where regions are usually urbanized. 
The experiments included in this paper showed how to use simulations to study realistic problems in a geothermal exploration context. These modeling routines are very useful for analyzing how significant can be the presence of metallic infrastructure, and how they could be used/exploited as a tool instead of being considered inconvenient during the exploration process. Based on our modeling results, we conclude that steel-cased wells can be used to amplify the EM field produced by vertical sources. More concretely, to improve signal-ration responses on the casing bottom (similar conclusions to that described in [16], [53]).

Regarding computational implementation, our parallel modeling routine can generate synthetic EM data that are almost identical with respect to real data measurements in land CSEM contexts (until now, it has only been successfully validated in marine environments). Our previously published adaptivemeshing technique has been validated in land contexts and, in particular, to incorporate small structures (e.g., metallic well casing) into the full 3-D modeling routine. Our meshing strategy proved to be capable of dealing with variable resolution discretizations (from kilometers to meters) and realistic physical parameters (e.g., resistivity). Also, a convergence test has been demonstrated that our adapted meshing technique can provide acceptable error levels while avoiding excessive refinement in the whole computational domain. Nevertheless, numerical accuracy and computational performance (e.g., runtime and memory consumption) depend on the chosen FE piece-wise order and input model. Numerical results show that PETGEM code features satisfy modeling requirements of realistic land CSEM setups. Latter is important because although the geological problem may, in some cases, be 2$\mathrm{D}$, to incorporate small infrastructures, a 3-D modeler is needed. We believe that our open-source 3-D CSEM modeler and experiments in the Vallès region will prove useful for geophysicists interested in arbitrary land 3-D CSEM modeling.

Our future research aims to study non-solid metallic casings and their impact on EM fields and discretization demands. Also, we intend to perform simulations for models that include Vertical Transverse Isotropic (VTI). Hopefully, this analysis will provide an in-depth understanding of electromagnetic modeling's pros and cons when steel-cased wells are present.

\section{CODE AVAILABILITY}

The PETGEM code is freely available at the home page (petgem.bsc.es), at the PyPI repository (pypi.org/project/petgem), at the GitHub site (github.com/ocastilloreyes/petgem), or by requesting it from the author (octavio.castillo@bsc.es). In all cases, the code is supplied to ease the immediate execution on Linux platforms. User's manual and technical documentation (developer's guide) are provided in the PETGEM archive.

\section{ACKNOWLEDGMENT}

This work was been developed in the frame of GEOURBAN project (PCI2018-092943 and PCI2018-093186). This work has received also partial funding from the $E u$ ropean Union's Horizon 2020 programme under the Marie
Sklodowska-Curie grant agreement $\mathrm{N}^{\circ}$ 777778. Also, the research leading to these results has received funding from the European Union's Horizon 2020 programme, grant agreement $\mathrm{N}^{\circ}$ 828947, and from the Mexican Department of Energy, CONACYT-SENER Hidrocarburos grant agreement $\mathrm{N}^{\circ}$ B-S-69926. Furthermore, this project has been $65 \%$ cofinanced by the European Regional Development Fund (ERDF) through the Interreg V-A Spain-France-Andorra program (POCTEFA2014-2020). POCTEFA aims to reinforce the economic and social integration of the French-Spanish-Andorran border. Its support is focused on developing economic, social and environmental cross-border activities through joint strategies favouring sustainable territorial development.

The authors would like to thank the Editors-in-Chief and to both reviewers for their valuable comments and suggestions that helped to improve the manuscript's quality. This work benefitted from the valuable suggestions, comments, and proofreading of Dr. Josep de la Puente (BSC).

\section{REFERENCES}

[1] G. Newman and D. Alumbaugh, "Three-dimensional massively parallel electromagnetic inversion-I. theory," Geophysical journal international, vol. 128, no. 2, pp. 345-354, 1997.

[2] T. Eidesmo, S. Ellingsrud, L. MacGregor, S. Constable, M. Sinha, S. Johansen, F. Kong, and H. Westerdahl, "Sea bed logging (SBL), a new method for remote and direct identification of hydrocarbon filled layers in deepwater areas," First break, vol. 20, no. 3, pp. 144-152, 2002.

[3] D. B. Avdeev, "Three-dimensional electromagnetic modelling and inversion from theory to application," Surveys in Geophysics, vol. 26, no. 6, pp. 767-799, 2005.

[4] S. Constable, "Marine electromagnetic methods-A new tool for offshore exploration," The Leading Edge, vol. 25, no. 4, pp. 438-444, 2006.

[5] L. J. Srnka, J. J. Carazzone, M. S. Ephron, and E. A. Eriksen, "Remote reservoir resistivity mapping," The Leading Edge, vol. 25, no. 8, pp. 972-975, 2006.

[6] S. Constable, "Ten years of marine CSEM for hydrocarbon exploration," Geophysics, vol. 75, no. 5, pp. 75A67-75A81, 2010.

[7] R.-U. Börner, "Numerical modelling in geo-electromagnetics: Advances and challenges," Surveys in Geophysics, vol. 31, no. 2, pp. 225-245, 2010.

[8] O. Castillo-Reyes, J. de la Puente, and J. M. Cela, "PETGEM: A parallel code for 3D CSEM forward modeling using edge finite elements," Computers \& Geosciences, vol. 119, pp. 123 - 136, 2018. [Online]. Available: http://www.sciencedirect.com/science/article/ pii/S0098300418301365

[9] O. Castillo-Reyes, J. de la Puente, L. E. García-Castillo, and J. M. Cela, "Parallel 3-D marine controlled-source electromagnetic modelling using high-order tetrahedral Nédélec elements," Geophysical Journal International, vol. 219, no. 1, pp. 39-65, 06 2019. [Online]. Available: https://doi.org/10.1093/gji/ggz285

[10] C. M. B. Nunes and C. Régis, "GEMM3D: An Edge Finite Element program for 3D modeling of electromagnetic fields and sensitivities for geophysical applications," Computers \& Geosciences, p. 104477, 2020.

[11] A. Orange, K. Key, and S. Constable, "The feasibility of reservoir monitoring using time-lapse marine CSEM," Geophysics, vol. 74, no. 2, pp. F21-F29, 2009

[12] M. Wirianto, W. Mulder, and E. Slob, "A feasibility study of land CSEM reservoir monitoring in a complex 3-d model," Geophysical Journal International, vol. 181, no. 2, pp. 741-755, 2010.

[13] J. Chen, G. M. Hoversten, D. Vasco, Y. Rubin, and Z. Hou, "A bayesian model for gas saturation estimation using marine seismic AVA and CSEM data," Geophysics, vol. 72, no. 2, pp. WA85-WA95, 2007.

[14] M. S. Zhdanov, M. Endo, N. Black, L. Spangler, S. Fairweather, A. Hibbs, G. Eiskamp, and R. Will, "Electromagnetic monitoring of $\mathrm{CO}_{2}$ sequestration in deep reservoirs," first break, vol. 31, no. 2, 2013. 
[15] E. Vilamajó, B. Rondeleux, P. Queralt, A. Marcuello, and J. Ledo, "A land controlled-source electromagnetic experiment using a deep vertical electric dipole: experimental settings, processing, and first data interpretation," Geophysical Prospecting, vol. 63, no. 6, pp. 1527-1540, 2015.

[16] E. Vilamajó, V. Puzyrev, P. Queralt, A. Marcuello, and J. Ledo, "Study of the casing effect on borehole-to-surface onshore CSEM," in 78th EAGE Conference and Exhibition 2016, vol. 2016, no. 1. European Association of Geoscientists \& Engineers, 2016, pp. 1-5.

[17] J. Park, G. Sauvin, and M. Vöge, "2.5D inversion and joint interpretation of CSEM data at Sleipner $\mathrm{CO}_{2}$ storage," Energy Procedia, vol. 114, pp 3989-3996, 2017.

[18] S. Tveit, T. Mannseth, J. Park, G. Sauvin, and R. Agersborg, "Combining CSEM or gravity inversion with seismic AVO inversion, with application to monitoring of large-scale $\mathrm{CO}_{2}$ injection," Computational Geosciences, pp. 1-20, 2020.

[19] J. D. Kana, N. Djongyang, D. Raïdandi, P. N. Nouck, and A. Dadjé, "A review of geophysical methods for geothermal exploration," Renewable and Sustainable Energy Reviews, vol. 44, pp. 87-95, 2015.

[20] N. Coppo, M. Darnet, V. Harcouet-Menou, P. Wawrzyniak, A. Manzella F. Bretaudeau, G. Romano, D. Lagrou, and J.-F. Girard, "Characterization of Deep Geothermal Energy Resources in Low enthalpy sedimentary basins in Belgium using Electro-Magnetic Methods-CSEM and MT results," in European Geothermal Congress 2016, 2016.

[21] M. Darnet, P. Wawrzyniak, N. Coppo, S. Nielsson, E. Schill, and G. Fridleifsson, "Monitoring geothermal reservoir developments with the Controlled-Source Electro-Magnetic method-A calibration study on the Reykjanes geothermal field," Journal of Volcanology and Geothermal Research, 2018.

[22] B. O. Omisore, S. Jin, and O. Fayemi, "Numerical modelling of Borehole-Surface CSEM response of onshore gas hydrate deposit with higher order finite difference method," Journal of Applied Geophysics, vol. 174, p. 103968, 2020.

[23] S. Constable, K. Key, and L. Lewis, "Mapping offshore sedimentary structure using electromagnetic methods and terrain effects in marine magnetotelluric data," Geophysical Journal International, vol. 176, no. 2, pp. 431-442, 2009.

[24] K. Key, "Marine electromagnetic studies of seafloor resources and tectonics," Surveys in geophysics, vol. 33, no. 1, pp. 135-167, 2012.

[25] S. E. Johansen and P. T. Gabrielsen, "Interpretation of marine CSEM and marine MT data for hydrocarbon prospecting," in Petroleum Geoscience. Springer, 2015, pp. 515-544.

[26] E. Vilamajó, P. Queralt, J. Ledo, and A. Marcuello, "Feasibility of monitoring the Hontomín (Burgos, Spain) $\mathrm{CO}_{2}$ storage site using a deep EM source," Surveys in Geophysics, vol. 34, no. 4, pp. 441-461, 2013.

[27] R. Streich, "Controlled-source electromagnetic approaches for hydrocarbon exploration and monitoring on land," Surveys in geophysics, vol. 37, no. 1 , pp. 47-80, 2016.

[28] F. Kong, F. Roth, P. Olsen, and S. Stalheim, "Casing effects in the seato-borehole electromagnetic method," Geophysics, vol. 74, no. 5, pp. F77-F87, 2009.

[29] F. Frezza, L. Pajewski, C. Ponti, G. Schettini, and N. Tedeschi, "Electromagnetic scattering by a metallic cylinder buried in a lossy medium with the cylindrical-wave approach," IEEE Geoscience and Remote Sensing Letters, vol. 10, no. 1, pp. 179-183, 2013.

[30] _ - "Cylindrical-wave approach for electromagnetic scattering by subsurface metallic targets in a lossy medium," Journal of Applied Geophysics, vol. 97, pp. 55-59, 2013.

[31] N. H. Cuevas, "Analytical solutions of EM fields due to a dipolar source inside an infinite casing," Geophysics, vol. 79, no. 5, pp. E231-E241, 2014.

[32] E. S. Um, M. Commer, G. A. Newman, and G. M. Hoversten, "Finite element modelling of transient electromagnetic fields near steel-cased wells," Geophysical Journal International, vol. 202, no. 2, pp. 901-913, 2015.

[33] E. S. Um, J. Kim, and M. Wilt, "3D borehole-to-surface and surface electromagnetic modeling and inversion in the presence of steel infrastructure," Geophysics, vol. 85, no. 5, pp. 1-54, 2020.

[34] D. Colombo and G. W. McNeice, "Quantifying surface-to-reservoir electromagnetics for waterflood monitoring in a Saudi Arabian carbonate reservoir," Geophysics, vol. 78, no. 6, pp. E281-E297, 2013.

[35] D. Colombo, G. McNeice, N. Cuevas, M. Pezzoli et al., "Surface to borehole electromagnetics for 3D waterflood monitoring: Results from first field deployment," in SPE Annual Technical Conference and Exhibition. Society of Petroleum Engineers, 2018.
[36] A. V. Grayver, R. Streich, and O. Ritter, "3D inversion and resolution analysis of land-based CSEM data from the ketzin $\mathrm{CO}_{2}$ storage formation," Geophysics, vol. 79, no. 2, pp. E101-E114, 2014.

[37] V. Puzyrev, E. Vilamajo, P. Queralt, J. Ledo, and A. Marcuello, "Threedimensional modeling of the casing effect in onshore controlled-source electromagnetic surveys," Surveys in Geophysics, vol. 38, no. 2, pp. 527-545, 2017

[38] M. J. Wilt, E. S. Um, E. Nichols, C. J. Weiss, G. Nieuwenhuis, and K. MacLennan, "Casing integrity mapping using top-casing electrodes and surface-based electromagnetic fields," Geophysics, vol. 85, no. 1, pp. E1-E13, 2020.

[39] F. Bretaudeau, S. Penz, N. Coppo, P. Wawrzyniak, and M. Darnet, “3D land CSEM inversion in noisy environment with a single transmiter inversion approach and application for geothermal water prospection," 2017.

[40] M. Darnet, N. Coppo, P. Reninger, P. Wawrzyniak, J.-F. Girard, and B. Bourgeois, "Airborne and land-based controlled-source electromagnetic surveying in challenging electromagnetic environments-application to geothermal exploration in a volcanic island," 2017.

[41] G. Hoversten, C. Schwarzbach, P. Belliveau, E. Haber, and R. Shekhtman, "Borehole to surface electromagnetic monitoring of hydraulic fractures," in 79th EAGE Conference and Exhibition 2017, vol. 2017, no. 1. European Association of Geoscientists \& Engineers, 2017, pp. $1-5$.

[42] E. S. Um, J. Kim, M. J. Wilt, M. Commer, and S.-S. Kim, "Finiteelement analysis of top-casing electric source method for imaging hydraulically active fracture zones," Geophysics, vol. 84, no. 1, pp. E23E35, 2019.

[43] G. Maggio, D. Kiyan, C. J. Bean, P. Queralt, R. Delhaye, C. Hogg, J. McAteer, and S. Blake, "The GEO-URBAN project: Exploring the Geothermal Potential of Dublin City using Electromagnetic and Passive Seismic Methods," 2019.

[44] H. Cai, X. Hu, J. Li, M. Endo, and B. Xiong, "Parallelized 3D CSEM modeling using edge-based finite element with total field formulation and unstructured mesh," Computers \& Geosciences, vol. 99, pp. 125134, 2017.

[45] I. Cantarero, "Deformation-fluid multiphase interaction within the fractures of the Barcelona Plain and the Vallès Basin: influence on fault rocks and diagenesis/interacció," Ph.D. dissertation, Universitat de Barcelona, 2013. [Online]. Available: https://www.tdx.cat/handle/ $10803 / 110412$

[46] G. Mitjanas, J. Ledo, G. Alias, A. Macau, P. Queralt, F. Bellmunt, L. Rivero, G. A, A. Marcuello, B. B, M. A, and F. S, "Integrated seismic ambient noise, magnetotellurics and gravity for the $2 \mathrm{D}$ interpretation of the Vallès Basin structure in the geothermal system of La Garriga-Samalús (NE Spain)," Geothermics, vol. 93, p. 102067, 2021. [Online]. Available: https://www.sciencedirect.com/science/article/pii/ S0375650521000274

[47] K. Key, "MARE2DEM: a 2-D inversion code for controlled-source electromagnetic and magnetotelluric data," Geophysical Journal International, vol. 207, no. 1, pp. 571-588, 2016.

[48] A. A. Kaufman, "The electrical field in a borehole with a casing," Geophysics, vol. 55, no. 1, pp. 29-38, 1990.

[49] K. Key, "1D inversion of multicomponent, multifrequency marine CSEM data: Methodology and synthetic studies for resolving thin resistive layers," Geophysics, vol. 74, no. 2, pp. F9-F20, 2009.

[50] C. Patzer, K. Tietze, and O. Ritter, "Steel-cased wells in 3-d controlled source em modelling," Geophysical Journal International, vol. 209, no. 2, pp. 813-826, 2017.

[51] N. H. Cuevas and M. Pezzoli, "On the effect of the metal casing in surface-borehole electromagnetic methods," Geophysics, vol. 83, no. 3, pp. E173-E187, 2018.

[52] M. Couchman, M. Everett, J. Charbonneau, and R. Robinson, "Steel casing effects on csem monitoring of unconventional reservoir stimulation," 2018.

[53] C. J. Kohnke, "Electromagnetic effects of steel-cased wells," Ph.D. dissertation, Colorado School of Mines, 2017. 